\title{
INVESTIGACIONES
}

\section{Escritura para la reflexión pedagógica: rol y función del Diario del Profesor en Formación en las Prácticas Iniciales}

\author{
Writing for pedagogycal reflection: role and function \\ of the in-training Teacher's diary in the initial practice
}

\section{Marcela Jarpa Azagra, ${ }^{a}$ Valentina Haas Prieto ${ }^{b}$ Damaris Collao Donoso ${ }^{a c}$}

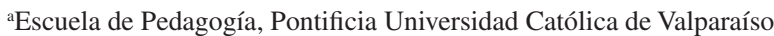

Correo electrónico: marcela.jarpa@pucv.cl

\author{
${ }^{b}$ Coordinadora de Prácticas de la carrera de Educación Básica, Pontificia Universidad Católica de Valparaíso \\ Correo electrónico: valentina.haas@pucv.cl \\ ${ }^{c}$ Correo electrónico: damaris.collao@pucv.cl
}

\begin{abstract}
RESUMEN
Esta investigación aborda la reflexión en las prácticas pedagógicas en la formación inicial docente mediante el análisis del Diario del Profesor en Formación. El objetivo es caracterizar la reflexión pedagógica que escriben los estudiantes de primer año en sus diarios, identificando el alcance de este y su función, el tipo de reflexión pedagógica y la naturaleza de ella. El enfoque metodológico proviene desde el Análisis del Discurso, el que permitió establecer categorías a priori sustentadas en la literatura especializada las que luego fueron consolidadas con el análisis de las unidades de significados proveniente de los diarios. Los principales hallazgos revelan nudos críticos en el desarrollo de la escritura para la reflexión pedagógica, entre los que se destaca la orientación fundamentalmente descriptiva y la elaboración de un tipo de reflexión superficial. La naturaleza de la reflexión es diversa, sin embargo, es posible identificar tópicos recurrentes los que presentan ciertos sesgos preliminares respecto de la identidad profesional del docente.
\end{abstract}

Palabras clave: escritura para reflexión pedagógica, diario del profesor, formación práctica.

\begin{abstract}
This research reviews the importance of reflection in teaching practices in initial teacher-training programs and focuses on the development of the "becoming teacher's diary" developed in the primary teaching career at Pontificia Universidad Catolica de Valparaiso. The aim is to characterize the pedagogical reflection written by freshmen in their diaries, identifying the use made of it, kind and nature of the pedagogical reflection. The methodological approach is part of the interpretive paradigm, based on the Discourse Analysis, in which categories are established a priori (top down) supported in the literature on the subject, and the analysis consolidated categories a posteriori (button up), which emerged from the units of meaning provided by the analyzed texts, a technique known as inductive deductive. The total study sample corresponded to 50 diaries. The main findings of the study reveal critical points in the development of writing for pedagogical reflection, among which it stands out the mainly descriptive orientation and a type of reflection that does not reach deep levels. Likewise, the nature of reflection is different, however, it is possible to identify recurring topics that are presented throughout the day, but have preliminary biases regarding the teacher's professional identity.
\end{abstract}

Key words: writing for pedagogical reflection, teacher's diary, Initial teacher training. 


\section{INTRODUCCIÓN}

Las diversas investigaciones en torno a la reflexión pedagógica en la formación inicial y continua de docentes (Ershler, 2003; Korthagen, 2010) abordan una perspectiva crítica de las acciones que llevan a cabo los maestros en sus prácticas pedagógicas, presentando diversos modelos y tipologías que dan cuenta de este tipo de reflexión.

La contribución fundamental de este trabajo apunta hacia otro ámbito de investigación vinculado al análisis de la escritura para la reflexión pedagógica desde la perspectiva del Análisis del Discurso y la Teoría del Género (Bathia, 2004; Swales, 2000), pues creemos que a partir de la identificación y descripción de determinados géneros discursivos que se utilizan para el desarrollo de la reflexión pedagógica es posible sistematizar los rasgos prototipos de esta y así andamiar, modelar y graduar su desarrollo como una competencia esencial a lo largo de la formación inicial docente. Al respecto, existen diversos géneros que reúnen la producción escrita de la reflexión pedagógica que se realiza a partir de la práctica docente, como por ejemplo: los diarios, registros etnográficos, portafolios, bitácoras, entre otros. Estos textos exigen para el escritor (estudiante) una demanda cognitiva mayor que no solo implica registrar su experiencia, sino que además darle coherencia y sentido a lo que presenta. Si a esto se suma el desafío de reflexionar respecto de un tópico determinado (la clase realizada, la conducta de los alumnos al interior del aula, formas de enfrentar un conflicto, los resultados de una evaluación aplicada) la tarea se vuelve altamente compleja.

En la carrera de Educación Básica de la Pontificia Universidad Católica de Valparaíso (Chile), los estudiantes de primer año asisten a Práctica Inicial y deben escribir un registro de la observación y experiencia vivida en las aulas escolares. Posteriormente, deben sistematizar esta información en el Diario del Profesor en Formación y asignarle una orientación fundamentalmente reflexiva. Al respecto, los diarios presentan diversas dificultades al momento de evaluarlos, pues aunque existe una pauta para ello (conocida por los estudiantes) la calidad de sus escritos está muy descendida. En primer lugar, los textos presentan problemas de redacción que hace que algunos de ellos sean ilegibles; en segundo lugar, no es posible distinguir una estructura retórica que pueda recoger y sistematizar la potencialidad de la experiencia de práctica; en tercer lugar, no se evidencia una reflexión pedagógica que permita identificar aspectos conceptuales que orienten un análisis más acabado de la experiencia de aula.

Bajo este contexto, surgió la necesidad de describir las características fundamentales de los diarios, considerando ciertos criterios de análisis que permitieran, por una parte, diagnosticar el alcance del uso de estos y su función para, posteriormente, poder modelar la escritura de estos textos con foco en el desarrollo de la reflexión pedagógica. Los criterios considerados para el análisis fueron objetivo del diario, naturaleza, nivel y tipo de escritura de la reflexión pedagógica.

\section{MARCO DE REFERENCIA}

\subsection{EL ROL DE LAS PRÁCTICAS INICIALES EN LA FORMACIÓN DOCENTE}

En el marco de la políticas públicas que se han desarrollado en Chile a partir del año 1997, el Ministerio de Educación chileno (MINEDUC) planteó el Programa de Fortalecimiento 
de la Formación Inicial Docente (FFID), el que reconoció la importancia de la práctica como eje articulador de la formación inicial docente (Ávalos, 2002). De esta manera, la configuración curricular de los planes de estudios de las carreras de pedagogías, a partir de entonces, sitúan a la formación práctica como una instancia fundamental que debe propiciar la articulación entre la teoría y la práctica, para permitir una aproximación gradual de los estudiantes al trabajo profesional y, al mismo tiempo, a la construcción e internalización del rol docente (Haas, 2011).

Las prácticas constituyen así una oportunidad en la que profesores en formación transfieren los conocimientos teóricos aprendidos hacia escenarios y contextos educativos reales, lo que permite un aprendizaje experiencial que favorece el desarrollo de un pensamiento reflexivo respecto del propio ejercicio docente (Cochran-Smith \& Lyte, 2009; Schön, 1998; Zabalza, 2011). En este sentido, la práctica adquiere una doble significación: por una parte, es un ejercicio de transición identitaria de inmersión a la profesión (Correa, 2011) y, por otro, un espacio de socialización profesional (Barquín, 2002; Lindgren, 2005; Tardif, 2004), donde el estudiante de pedagogía comienza a conocer las formas como se comunican, interactúan y desenvuelven los profesores en su ejercicio profesional.

Las prácticas iniciales constituyen un espacio protegido y el punto de encuentro entre el mundo profesional y el universitario (Correa, 2011) donde los estudiantes están llamados a contrastar sus aptitudes, actitudes y conocimientos en un ejercicio de empoderamiento y reconocimiento de lo que constituirá su futuro profesional. En síntesis, la práctica pedagógica como eje sobre el que se articula la formación inicial docente es una oportunidad para que los futuros profesores movilicen sus competencias en un marco de aprendizaje que es multidimensional (Haas, 2011). En él se contrastan sus saberes académicos, competencias conceptuales, procedimentales y actitudinales todo ello al realizar el contraste con la realidad en la que les corresponde intervenir. Esto se consolida como un verdadero ejercicio de articulación que los acerca a su inserción profesional.

\subsection{EL PAPEL DE LA REFLEXIÓN EN LAS PRÁCTICAS PEDAGÓGICAS}

Un modelo de formación basado en la enseñanza reflexiva debería desarrollar la capacidad de reflexión de los futuros profesores como herramienta para repensar su propia enseñanza, su crecimiento personal y el desarrollo profesional (Zeichner, 1986). La idea del profesor como un profesional reflexivo supone una reconceptualización de la enseñanza y, por supuesto, de la práctica docente, pues esta se convierte en un esquema de acción y en hipótesis de su trabajo. Al respecto, Tezanos (2007) destaca el papel de la reflexión en la construcción del saber pedagógico y señala que esta debe ser abordada desde dos perspectivas: primero, como una actividad mental-psicológica del ser humano y, segundo, como praxis social.

Esta doble aproximación permite comprender y proyectar la forma como enseñar la reflexión pedagógica en la formación docente, pues sin duda es una habilidad que necesita ser modelada. Por una parte, hay que reconocer y valorar la complejidad del ejercicio reflexivo, pues en él convergen diversas operaciones cognitivas que movilizan recursos atencionales, motivacionales, memorísticos, interpretativos, entre otros. Gracias a ellos es posible construir un análisis profundo que pone en juego al propio sujeto y al mundo que lo rodea.

Por otra parte, abordar la reflexión pedagógica como una práctica social implica reflejar el propio mundo, reflexionar sobre él y reflexionar sobre sí mismo; acciones que están en el centro del proceso en el que se forma el sujeto y, por lo tanto, gracias a esto es 
posible construir conocimiento pedagógico y adquirir conciencia del devenir histórico del rol del profesor en la sociedad.

Esta doble mirada de la reflexión pedagógica permite identificar tipos de reflexión, como por ejemplo, la propuesta de Van Manen (1977) quien reconoce tres niveles. El primero, lo denomina reflexión técnica, pues el foco está puesto solo en la eficiencia y eficacia de los medios utilizados para alcanzar ciertos fines, por lo mismo, esta reflexión es más hermética y no permite una perspectiva crítica ni modificación alguna. El segundo nivel, lo constituye la reflexión práctica, que permite un examen más amplio no solo de los medios, sino que también, de los objetivos y supuestos sobre los que se obtienen los resultados. De la misma manera, se reconoce que existe una negociación de los significados que se ponen en juego en el proceso de aprendizaje y, por lo mismo, el lenguaje adquiere una importancia relevante para este nivel. Finalmente, la reflexión crítica, tercer nivel, incorpora los dos anteriores, pero además implica criterios éticos y morales relacionados con una perspectiva histórica, social y política de los fenómenos implicados.

Por su parte, Hatton y Smith (1995) en un estudio realizado en la Universidad de Sydney a estudiantes de pedagogía de último año, reconoce cuatro tipos de escritura para la reflexión pedagógica. La primera la denominan escritura descriptiva, que no es considerada reflexión propiamente tal, pero que está presente en los textos que escriben los estudiantes y que más bien hacen referencia a la descripción de los eventos que ocurren en contextos escolares o bien a la reproducción de ciertos temas provenientes de la literatura especializada. Un segundo tipo es la reflexión descriptiva, la que entrega razones o argumentos respecto de los juicios emitidos, ya sea con fundamentos propios o bien extraídos y comentados desde ciertos autores; el tercer tipo es la reflexión dialógica, que pone en evidencia la exploración interna de las posibles razones que movilizan la toma de decisiones de las acciones implementadas en determinada situación y, por último, la reflexión crítica, que expone y analiza los contextos históricos y culturales implicados.

Recogiendo los postulados de Firdyiwek y Scida (2014), consideramos que la reflexión pedagógica es un vehículo de aprendizaje capaz de proponer nuevas técnicas de enseñanza, por esta razón, el proceso reflexivo del profesor debe ser una tarea sistemática en el desarrollo de su labor docente y no limitarse a los momentos de evaluación. Es mediante un proceso continuo que se enriquece la práctica pedagógica y las estructuras de conocimiento, creencias y valores tanto de los futuros docentes como de aquellos que están en ejercicio, por ello, la reflexión debe ser una tarea constante en el desarrollo profesional del profesor.

De acuerdo con lo anterior, el desarrollo de una enseñanza reflexiva implica abordar una teoría de la reflexión que considere el objeto de la reflexión, el proceso reflexivo y las actitudes reflexivas, de lo contrario, al no sistematizar y desarrollar un pensamiento reflexivo se reduce a un ejercicio intuitivo sin anclaje racional y real. Al respecto, se sugieren ciertas estrategias para propiciar una formación reflexiva, destacando, por ejemplo, los proyectos de investigación acción, estudios de caso y estudios etnográficos, experiencias de supervisión prácticas, tareas curriculares y los diarios de prácticas.

Con el fin de instaurar el ejercicio reflexivo en el quehacer del profesor, diversos autores destacan la escritura de diarios de práctica como una metodología capaz de dar cuenta de los procesos reflexivos de los docentes (Boud, 2001; Liston \& Zeichner, 1990; McGuire, Lay \& Peters, 2009; Prestridge, 2014; Scale, 2013). En el ámbito de las prácticas pedagógicas, la escritura se erige como un proceso que da cuenta de la naturaleza de la reflexión pedagógica y, junto con ello, ayuda a construir la identidad del futuro profesional 
(Nguyen et al., 2014). Por lo anterior, el producto de este proceso reflexivo se convierte en un insumo que impacta en el aprendizaje del docente, y le permite elaborar sus propias teorías sobre el aprendizaje y la enseñanza.

\subsection{EL DIARIO DEL PROFESOR EN FORMACIÓN: UNA ESTRATEGIA PARA POTENCIAR LA REFLEXIÓN PEDAGÓGICA}

Una de las aproximaciones conceptuales al diario del profesor es abordarlo como un documento de registro escrito donde los profesores recogen sus impresiones sobre lo que va sucediendo en sus clases (Zabalza, 2004), con lo cual constituye un instrumento de formación que facilita la implicación y despliega la introspección. También es posible considerarlo como un instrumento de investigación que desarrolla la observación y la autoobservación, que permite recoger datos de diferente índole $\mathrm{y}$, por lo tanto, es una herramienta de análisis del pensamiento reflexivo de profesores tanto en formación como en ejercicio (Latorre, 1996; Porlán \& Martin, 1993).

Sin embargo, no todos los diarios son similares y, por lo tanto, es posible caracterizarlos a partir de ciertos criterios diferenciadores: el contenido, el proceso de recogida, redacción y análisis de la información y la función de este.

De este modo, desde el contenido es posible identificar una amplia variedad de temáticas, pues se escribe de todo aquello que es factible de destacar. En este punto es necesario señalar que creemos que para que la reflexión sea efectiva y trascienda la formación del docente se deben predefinir ciertos tópicos y así intencionar la observación y registro. El autor es otro aspecto importante, pues pueden ser variados: profesores de aula, profesores en formación, profesores noveles o bien mentores; cualquiera sea el narrador la perspectiva cambia y, por lo tanto, nos encontramos con relatos, tópicos y niveles de reflexión muy diversos. Por su parte, el marco espacial que ancla el relato, por lo general, es el aula o la clase, no obstante, también es posible abordar otros escenarios.

En cuanto al proceso de recogida de información, la periodicidad es un aspecto determinante, pues es importante resguardar cierta línea de continuidad en la recogida y redacción de las narraciones. Desde el punto de vista metodológico, los diarios delimitan enfoques o líneas de investigación basadas en documentos personales, que entregan información, por ejemplo, de trayectorias profesionales, ciclos vitales o itinerarios formativos, entre otros (Zabalza, 2004).

La función narrativa del diario (Holly, 1989) también proporciona diferencias en su constitución y alcance. Así pues, algunos diarios asumen una narración más periodística (journalistic writing) que pone el foco en la descripción de los acontecimientos, sería lo que Hatton y Smith (1995) denominan como escritura descriptiva. En cambio otros, son más analíticos (analytical writing) y fijan diversas dimensiones de observación, especificando ciertos aspectos que pueden ser relevantes según requerimientos predefinidos o aquellos de corte más evaluativo (evaluative writing), donde el foco está en los juicios y valoraciones emitidos. También están los que presentan una escritura más subjetiva y con foco en las emociones (therapeutic writing), cuyo sentido principal es descargar tensiones o que el contenido del diario se vuelque sobre sí mismo (introspective writing).

Cualquiera sea el tipo, el impacto formativo de los diarios, según Zabalza (2004), tiene múltiples potencialidades: en primer lugar, permite a los profesores revisar su mundo personal, poniendo énfasis en aquellas experiencias que han marcado su ejercicio 
profesional; también permite explicitar los propios dilemas, lo que permite enfocar el análisis y la reflexión en situaciones de conflicto o dialécticas; por otro lado, permite la evaluación y reajuste de procesos didácticos que se llevan a cabo y, finalmente, es una fuente para el desarrollo profesional permanente, ya que propicia un proceso de revisión y análisis de la propia práctica, instalando círculos de mejora permanente en el ejercicio docente.

Es importante destacar que el sentido básico del diario es convertirse en un espacio narrativo de los pensamientos de los profesores, en otras palabras, es un recurso expresivo que potencia la escritura, la reflexión y permite organizar el pensamiento desde un sentido histórico, integrando lo expresivo y referencial en un mismo relato.

\section{LA INVESTIGACIÓN}

\subsection{ENFOQUE METODOLÓGICO}

Las orientaciones metodológicas para el desarrollo de esta investigación provienen desde el Análisis del Discurso, el que se inscribe dentro del paradigma cualitativo y desde una perspectiva interpretativa (Valles, 2000). De esta forma, el análisis se sitúa en el orden de los significados y las acciones que estos determinan en el contexto estudiado.

El objeto de estudio es el texto, es decir, cada uno de los diarios que escribieron los estudiantes de primer año de la carrera de Educación Básica de la Pontificia Universidad Católica de Valparaíso en su práctica inicial. En cuanto a la unidad de análisis, esta corresponde a los segmentos textuales asociados a un determinado propósito comunicativo y que permite configurar la organización retórica de este género discursivo.

El análisis de los textos del corpus se aborda desde una doble dimensión. Por una parte, es de orden deductivo, pues se establecieron categorías a priori que correspondieron al objetivo, la naturaleza y el tipo de reflexión pedagógica. Par ello se procedió a buscar en la literatura especializada los aportes conceptuales que dieran cuenta de estos aspectos y asî buscar en los textos la información referida a estas categorías. Por otro lado, la dimensión inductiva, se relaciona con los significados emergentes asociados a estas categorías y que surgen de la interpretación de los diversos segmentos textuales. Así por ejemplo, para la categoría que daba cuenta del objetivo del diario, emergieron dos ámbitos de significación que serán explicados más adelante.

Este tipo de técnica de análisis (deductiva-inductiva) nos permite alcanzar el objetivo de esta investigación y dar respuesta a las preguntas que guiaron este estudio.

\subsection{PREGUNTA DE INVESTIGACIÓN}

¿Qué características presenta la reflexión pedagógica escrita de los estudiantes de primer año de Educación Básica de la Pontificia Universidad Católica de Valparaíso cuando elaboran el Diario del Profesor en Formación durante su Práctica Inicial?

A partir de ella se construyen las siguientes preguntas específicas:

- ¿qué objetivo presenta el Diario del Profesor en Formación?

- ¿qué tipo de reflexión se desarrolla en el Diario del Profesor en Formación?

- ¿cuál es la naturaleza de la reflexión en el Diario del Profesor en Formación? 


\subsection{OBJETIVOS DE LA INVESTIGACIÓN}

\section{Objetivo General}

- Caracterizar la reflexión pedagógica que escriben los estudiantes de primer año de la carrera de Educación Básica de la PUCV mediante el análisis del Diario del Profesor en Formación.

\section{Objetivos Específicos}

- Identificar el objetivo del uso del diario en la formación pedagógica de la práctica inicial docente.

- Describir el tipo de reflexión pedagógica que promueve la escritura del Diario del Profesor en Formación.

- Describir la naturaleza de la reflexión pedagógica que promueve la escritura del Diario del Profesor en Formación.

\subsection{DISEÑO DE INVESTIGACIÓN}

Para caracterizar la reflexión pedagógica presente en el Diario del Profesor en Formación se consideró el método de análisis de segmentos textuales proveniente desde la Teoría del Género. La Tabla 1 detalla los principales aspectos de este diseño.

Tabla 1. Principales aspectos del diseño de la investigación

\begin{tabular}{|c|c|c|}
\hline Aspecto & Tipo & Descripción operacional \\
\hline Objeto de Estudio & Artefacto: diarios escritos & $\begin{array}{l}\text { Producto tangible que describe por es- } \\
\text { crito la observación de clases en la } \\
\text { práctica inicial }\end{array}$ \\
\hline Unidad de Análisis & Segmentos textuales del diario & $\begin{array}{l}\text { Conjunto de palabras con coherencia } \\
\text { local marcado por un punto seguido o } \\
\text { aparte }\end{array}$ \\
\hline Muestra & Muestreo intencionado & 50 diarios escritos \\
\hline \multirow{3}{*}{ Criterios de recolección } & Selección del escenario & $\begin{array}{l}\text { Carrera de Educación Básica Asigna- } \\
\text { tura de Práctica Inicial, ubicada en el } \\
\text { primer y segundo semestre de primer } \\
\text { año }\end{array}$ \\
\hline & Selección de participantes & Estudiantes de primer año \\
\hline & Determinación del momento & $\begin{array}{l}\text { Los diarios se recogen al finalizar el } \\
\text { primer semestre, pues se cuenta con el } \\
\text { registro total de las actividades descri- } \\
\text { tas durante las } 12 \text { sesiones asistidas a } \\
\text { los colegios }\end{array}$ \\
\hline
\end{tabular}




\subsection{PROCEDIMIENTOS DE ANÁLISIS DE LOS DATOS}

Para llevar a cabo el análisis de los diarios, se identificó como unidad de análisis los segmentos textuales. Esto implicó desarrollar dos procedimientos de análisis: el primero de carácter inductivo- analítico que implicó un análisis preliminar del corpus, a partir del cual emergieron categorías conceptuales a priori; el segundo, de carácter deductivo-analítico, con categorías conceptuales predefinidas y provenientes de la literatura especializada que permitiera clasificar los resultados preliminares. De la misma manera, es necesario señalar que el análisis de los datos fue emergente y recursivo, no obstante, es posible identificar tres etapas descritas a continuación en la Tabla 2.

Tabla 2. Descripción de las etapas y fases del análisis

\begin{tabular}{|c|c|}
\hline Etapas y fases del análisis & Descripción \\
\hline \multicolumn{2}{|l|}{ ETAPA 0: Conformación del corpus } \\
\hline $\begin{array}{l}\text { Fase 1: Codificación de cada uno de los } \\
\text { diarios }\end{array}$ & $\begin{array}{l}\text { Se codifican los diarios y las sesiones correspondientes } \\
\text { en cada uno }\end{array}$ \\
\hline $\begin{array}{l}\text { Fase 2: Digitalización de cada uno de los } \\
\text { diarios en formato PDF }\end{array}$ & $\begin{array}{l}\text { Se digitalizan los diarios y se construyen archivos digi- } \\
\text { tales para cada uno }\end{array}$ \\
\hline $\begin{array}{l}\text { Fase 3: Distribución de los diarios para } \\
\text { el análisis }\end{array}$ & $\begin{array}{l}\text { Se distribuyen los diarios para la lectura y análisis de } \\
\text { ellos entre los miembros del equipo. }\end{array}$ \\
\hline $\begin{array}{l}\text { Fase 4: Configuración de unidades para } \\
\text { el análisis }\end{array}$ & $\begin{array}{l}\text { Se definen dos unidades de análisis una macro y otra } \\
\text { micro: } \\
\text { La unidad macro corresponde a cada sesión de observa- } \\
\text { ción de clases registrada en el diario ( } 12 \text { sesiones) } \\
\text { La unidad micro corresponde al segmento textual de } \\
\text { cada sesión registrada desde donde se relevarán las ca- } \\
\text { tegorías conceptuales }\end{array}$ \\
\hline Fase 5: Análisis preliminar & $\begin{array}{l}\text { Se dividió el corpus total de } 50 \text { diarios en dos grupos a } \\
\text { cargo de los asistentes de investigación. Cada uno debió } \\
\text { analizar } 25 \text { diarios }\end{array}$ \\
\hline $\begin{array}{l}\text { Fase 6: Conformación de equipo para } \\
\text { evaluación de pares y juicio de expertos }\end{array}$ & $\begin{array}{l}\text { Se dividió el corpus total de } 50 \text { diarios en tres grupos, } \\
\text { cada uno a cargo de un investigador: } 2 \text { a cargo de } 16 \text { y } \\
\text { uno a cargo de } 18 \text {. }\end{array}$ \\
\hline \multicolumn{2}{|c|}{ ETAPA 1: Configuración del marco para el análisis inductivo-analítico } \\
\hline $\begin{array}{l}\text { Fase 1.1: Identificación de conceptos a } \\
\text { priori }\end{array}$ & $\begin{array}{l}\text { A partir de una lectura inicial se identifican categorías } \\
\text { conceptuales emergentes que den cuenta de aspectos } \\
\text { generales de los diarios. }\end{array}$ \\
\hline Fase 1.2: Primera triangulación & $\begin{array}{l}\text { Se revisan y ajustan las categorías conceptuales de am- } \\
\text { bos grupos de análisis. }\end{array}$ \\
\hline Fase 1.3: Producción de categorías & $\begin{array}{l}\text { Se asignan y definen las categorías conceptuales prelimi- } \\
\text { nares para la organización textual y los temas abordados. }\end{array}$ \\
\hline
\end{tabular}




\begin{tabular}{|c|c|}
\hline Fase 1.4: Diagnóstico preliminar & $\begin{array}{l}\text { A partir de la categorías antes descritas se identifican } \\
\text { las características emergentes que dan origen a un diag- } \\
\text { nóstico preliminar respecto de la estructura y función } \\
\text { del diario }\end{array}$ \\
\hline \multicolumn{2}{|c|}{ ETAPA 2: Configuración del marco para la clasificación deductiva-analítica } \\
\hline $\begin{array}{lrrr}\text { Fase 2.1: } & \text { Ajuste } & \text { de } & \text { categorías } \\
\text { conceptuales } & \text { desde } & \text { la } & \text { literatura } \\
\text { especializada } & & & \end{array}$ & $\begin{array}{l}\text { Se ajustan las categorías conceptuales preliminares con } \\
\text { las definidas desde la literatura especializada }\end{array}$ \\
\hline $\begin{array}{l}\text { Fase 2.2: Clasificación de categorías } \\
\text { conceptuales de reflexión pedagógica }\end{array}$ & $\begin{array}{l}\text { A partir de la descripción preliminar de los temas de } \\
\text { reflexión, se clasifica el tipo de reflexión y la naturaleza } \\
\text { de esta según autores revisados. }\end{array}$ \\
\hline \multicolumn{2}{|c|}{$\begin{array}{l}\text { ETAPA 3: Validación de los resultados. Se realiza un proceso de triangulación para establecer la } \\
\text { validez de los resultados }\end{array}$} \\
\hline Fase 3.1 Comprobación de miembros & $\begin{array}{l}\text { Evaluación del proceso de análisis en cada etapa por } \\
\text { parte del equipo de investigación. }\end{array}$ \\
\hline
\end{tabular}

Fuente: elaboración propia.

\section{RESULTADOS Y DISCUSIÓN}

Como resultado de esta investigación se obtuvo un diagnóstico de las principales características de la reflexión pedagógica presente en los diarios escritos por estudiantes de primer año de la carrera de Educación Básica en su práctica inicial. Esto es coherente con cada uno de los objetivos planteados al inicio de la investigación y cuyos resultados se presentan organizados en la Figura 1. Para sistematizar los resultados y realizar un análisis en profundidad se relevaron tres dimensiones. La primera da cuenta del objetivo del diario a partir de dos aspectos intrínsecos a él: por una parte, el propósito comunicativo del diario y, por otra, el objetivo de aprendizaje del mismo. La segunda dimensión analiza el tipo de reflexión que emerge del análisis de los segmentos textuales de los diarios y con ello también es posible identificar el tipo de escritura asociado. Finalmente, la tercera dimensión implica la naturaleza u objeto que motiva el análisis de la observación realizada en la práctica. 
Figura 1. Dimensiones del análisis para diagnóstico de la reflexión pedagógica

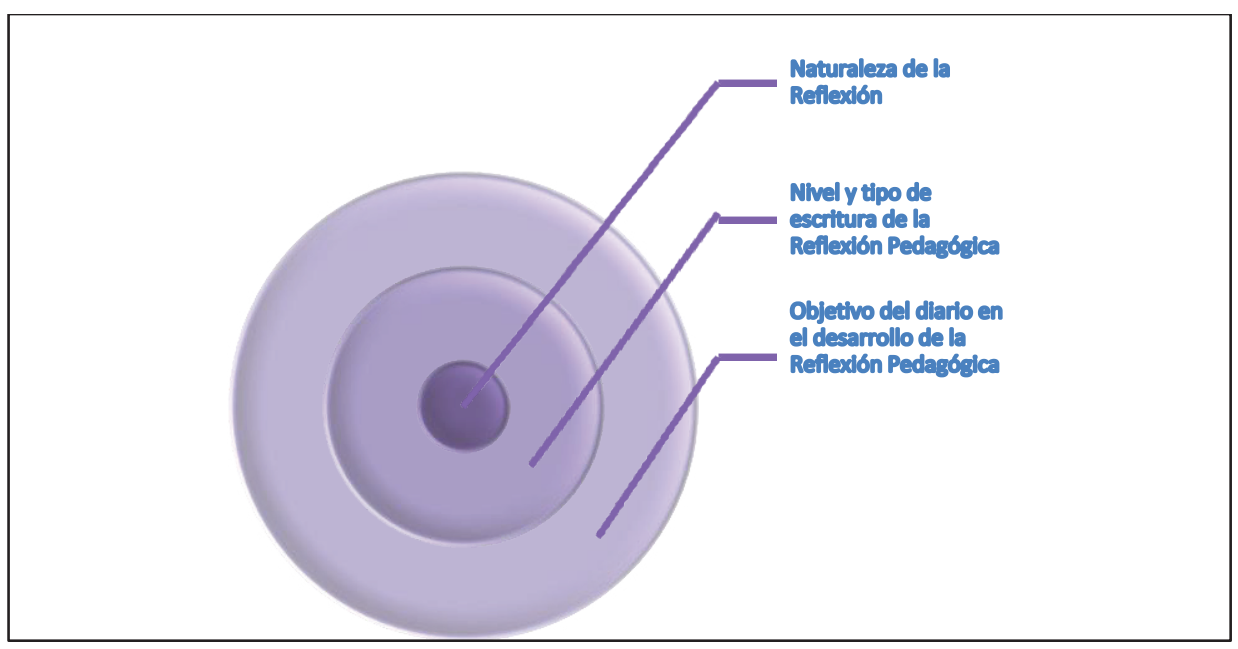

Fuente: elaboración propia.

A continuación, cada una de estas dimensiones es descrita en profundidad según los objetivos de investigación planteados.

\subsection{OBJETIVO DEL DIARIO DEL PROFESOR EN FORMACIÓN PARA EL DESARROLLO DE LA REFLEXIÓN PEDAGÓGICA}

Si bien los diarios de los profesores no son en sí mismos una novedad, pues se han utilizado con distintas finalidades y en diversas etapas de la carrera docente (preparar itinerarios didácticos, control de las clases o bien registro anecdótico y subjetivo de la experiencia docente), en la carrera de Educación Básica de la PUCV el uso del diario adquiere ciertas particularidades, pues es un instrumento de evaluación del proceso formativo en el ámbito de la práctica inicial y, por lo mismo, debiera dar cuenta de un resultado de aprendizaje que esté directamente vinculado con el desarrollo de la reflexión pedagógica.

Para poder desarrollar este aspecto relevamos dos criterios: por una parte, identificar el propósito comunicativo del diario y, por otra, el objetivo de aprendizaje. De esta forma, es posible reconocer que el propósito comunicativo del diario era describir las observaciones de las clases, registrando situaciones dialécticas o conflictivas que se producen al interior del aula o bien durante los recreos. El resultado de esto son relatos cargados de un fuerte componente emocional donde se privilegia la descripción de sentimientos, interpretaciones, conjeturas o explicaciones respecto de un determinado acontecimiento. De la misma manera, es frecuente encontrar extensas narraciones, algunas con carácter literario, que ejemplifican o describen los contextos donde suceden dichos acontecimientos. La Imagen 1 ejemplifica lo anteriormente planteado. 


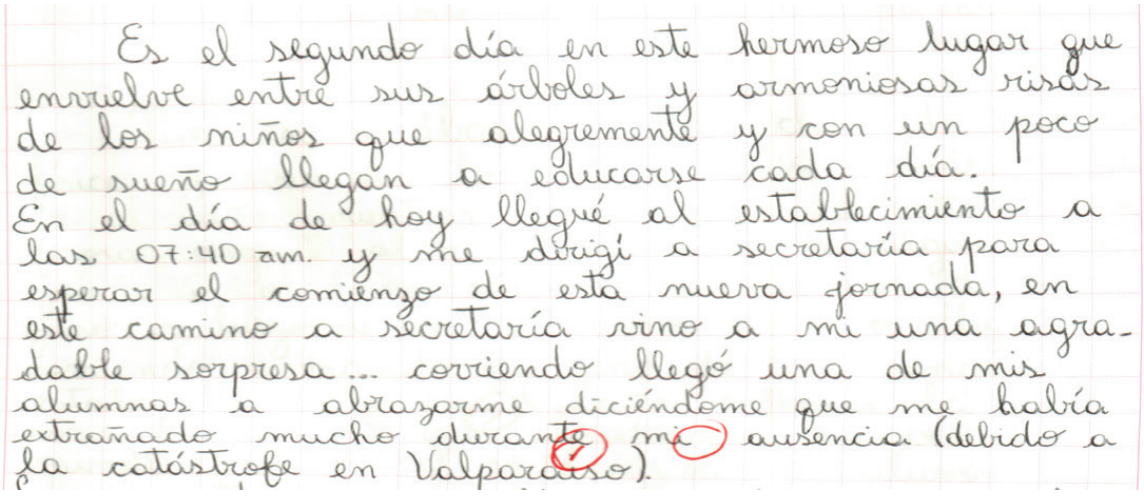

Fuente: elaboración propia.

Para analizar el objetivo de aprendizaje del diario consideramos el Modelo de Objetivos de Aprendizaje de Anderson y Krathwohl (2001), pues esta propuesta considera dos dimensiones: una en relación al conocimiento movilizado en el objetivo de aprendizaje y otra que da cuenta de los procesos cognitivos implicados. Es así como desde la primera dimensión, el conocimiento que se moviliza en los aprendices corresponde a uno de tipo factual, ya que lo que se registra en el diario son detalles y aspectos específicos que fueron observados durante la visita a la sala de clases o durante el recreo. Este tipo de conocimiento obedece a un estadio de desarrollo más bien concreto, pues solo se requiere conocer los elementos básicos para tener conocimiento de una disciplina o bien resolver problemas asociados a esta.

En cuanto a la dimensión de los procesos cognitivos, la escritura del diario involucra habilidades de los dos primeros niveles que son recuerdo y comprensión. La habilidad de recordar moviliza acciones que tienen relación con reconocer e identificar los diversos elementos que participan en la situación de aula que es descrita y considerada como relevante para comprender la dinámica de grupo y el nudo crítico que quiere ser expuesto. Por su parte, el proceso de comprensión pone en juego, fundamentalmente, la habilidad para ejemplificar estas mismas situaciones desde la realidad percibida por el estudiante en práctica inicial.

Por lo tanto, aun cuando el objetivo de aprendizaje solo aborda niveles básicos y concretos de conocimiento y de procesos cognitivos, es necesario modelar y acompañar las habilidades de escritura que el diario demanda. En otras palabras, generar actividades que permitan evaluar el nivel de descripción logrado, tanto en términos textuales como terminológicos. Esto debiera estar abordado en el diseño del programa del curso y en las pautas de evaluación de los productos esperados.

\subsection{TIPO DE REFLEXIÓN PEDAGÓGICA}

La literatura especializada presenta algunos tipos de reflexión pedagógica, según diversos criterios caracterizadores. En este caso, consideramos el nivel de reflexión (Van Manen, 1977) y el tipo de escritura (Hatton \& Smith, 1995; Holly, 1989). 
Al respecto, el diario del profesor en formación se caracteriza por presentar un nivel de reflexión pedagógica que se aproxima a racionalidad técnica (Van Manen, 1977), pues la atención se centra en los medios utilizados por los docentes en ejercicio para alcanzar ciertos fines pedagógicos, como puede ser la atención de los estudiantes, la buena conducta, la participación en clases, etc. Sin embargo, al ser una observación de aula que se enmarca en una práctica inicial temprana, los futuros profesores no cuentan con las herramientas y los conocimientos pedagógicos específicos para poder describir y analizar los recursos implicados. Esto hace que el nivel de reflexión sea superficial y general, carente de conceptualizaciones y explicaciones técnicas.

En cuanto al tipo de escritura reflexiva esta coincide con lo planteado por Hatton y Smith (1995), pues esta es descriptiva, casi como una narración periodística que pone el foco en el relato de los acontecimientos que suceden al interior del aula, por lo mismo, es frecuente encontrar el detalle de secuencias cronológicas por sobre secuencias explicativas y argumentativas. Es así como estos escritos reúnen fundamentalmente opiniones y percepciones personales respecto del quehacer de un profesor en ejercicio, el cual es fundamentalmente observado, poniendo atención a las habilidades y competencias, en cuanto medios o herramientas que despliega al interior del aula o fuera de ella, para desarrollar su clase. A continuación se presentan las principales características que este tipo de reflexión presenta en los diarios:

1. Se evidencia en la narrativa de los diarios una prevalencia de guiones centrados en aspectos emocionales, sobre cómo se sienten al momento de realizar una u otra tarea en la práctica, se deja de lado el análisis descriptivo y teórico de las situaciones vivenciadas y se pierde de vista la finalidad de la reflexión, que tal como señala Imbernón (2004), permite obtener las respuestas a las diversas problemáticas de la enseñanza, así como el desarrollar nuevas formas de comprensión de su propia intervención.

2. La escritura coherente y adecuada de los diarios está correlacionada con las competencias de entrada de los estudiantes de primer año. Es decir, aquellos alumnos que presentan competencias comunicativas descendidas (errores de ortografía y redacción) presentan mayores complejidades a la hora de expresar sus vivencias en el aula de práctica y obtienen menores ponderaciones de evaluación en sus narraciones diarias.

Este tipo de escritura y nivel de reflexión es considerado como básico y las interpretaciones e incluso las evaluaciones morales (en algunos casos) surgen a partir de las preocupaciones personales y experiencias previas que los estudiantes en práctica posean. En este sentido, el diario del profesor en formación es una herramienta útil para preparar a los futuros profesores al ingreso a la profesión, pues permite, en un estadio inicial, aproximarse al ejercicio docente a pequeña escala y en entornos controlados, lo que permite también identificar a priori algunos nudos críticos. De la misma forma, estos escritos revelan creencias o sesgos por parte de los futuros profesores, los que debieran ser modificados durante el proceso formativo. 


\subsection{NATURALEZA DE LA REFLEXIÓN PEDAGÓGICA}

En cuanto al tercer eje temático de la caracterización, la naturaleza de la reflexión pedagógica, este guarda relación con el objeto de análisis que es predominante en los diarios y que, por lo tanto, también corresponde al objeto que es descrito con mayor profundidad en los relatos. La Figura 2 sistematiza los hallazgos encontrados a partir del corpus de diarios.

Figura 2. Naturaleza de la reflexión pedagógica del Diario del Profesor en Formación

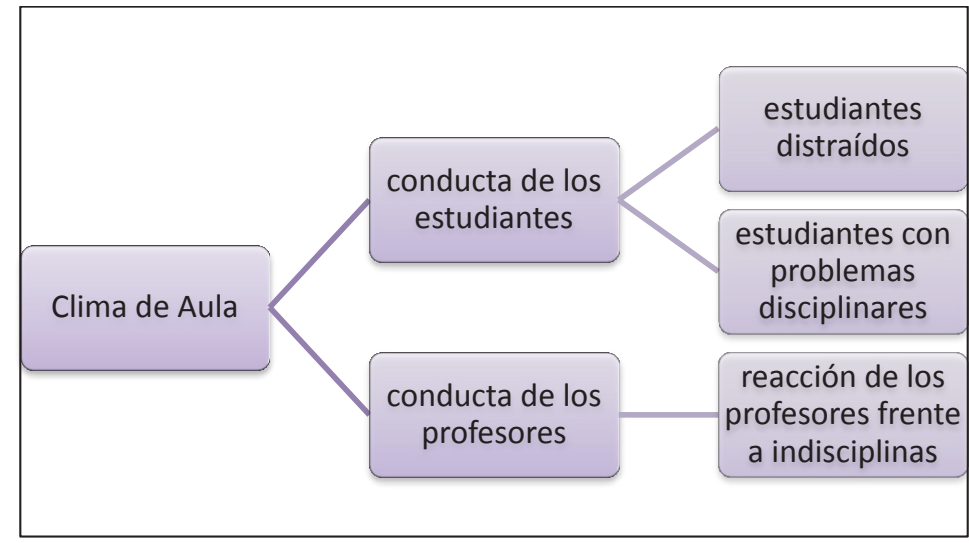

Fuente: elaboración propia.

En primer lugar, es necesario destacar que la naturaleza de la reflexión pedagógica de los estudiantes es, fundamentalmente, sobre el clima de aula y la forma como el docente lo encausa. Así pues, un primer aspecto que llama la atención y que es descrito en los diarios es el comportamiento de los escolares en dos ámbitos: uno respecto de la concentración y participación durante las clases y, otro, respecto de la conducta disciplinada o no que manifiestan frente a acciones del propio docente o de los compañeros.

En este sentido es frecuente encontrar extensas y detalladas narraciones de situaciones de conflicto al interior del aula, las que son descritas y en algunos casos enjuiciadas desde su propia experiencia y sin fundamentos o razones que permitan evidenciar una reflexión pedagógica o crítica de los contextos sociales, históricos o políticos que subyacen. Es aquí donde es posible evidenciar las creencias o representaciones sociales que traen consigo y que están marcadas por su propia experiencia como estudiantes y no con una perspectiva profesional.

Un segundo aspecto que es objeto de observación, es la conducta o la reacción de los docentes frente a las situaciones de conflicto. Particularmente, estos momentos son narrados con un carácter más literario, con énfasis en la tensión que experimentan sobre todo los niños y en muchos casos se sesga el actuar del docente mediante la presencia explicita de juicios o valoraciones positivas o negativas.

También es posible distinguir que las narraciones están sumergidas en declaraciones sobre aquello que anhelan ser o hacer cuando sean docentes y dejan de lado la reflexión 
y análisis de los hechos ocurridos al interior del aula como elementos que requieren conciencia reflexiva para su mejora.

La naturaleza de la reflexión pedagógica puede ser variada, y la profundidad y sistematicidad con que sea abordada va a depender de dos aspectos fundamentales: primero de las herramientas analíticas que se le entreguen al estudiante, pues estas le permitirán conocer y abordar diversas dimensiones del objeto. Esto significa que mientras más elementos teóricos y conceptuales domine mayor será su nivel de análisis.

En cuanto al segundo aspecto, este se relaciona con las orientaciones o directrices de la observación, es decir, enmarcar y delimitar el objeto de forma preliminar, pues esto le permitirá fijar su atención en él, realizando un seguimiento y profundización para poder comprenderlo en toda su dimensión.

\section{CONCLUSIONES Y PROYECCIONES}

Un primer aspecto a destacar es la importancia de generar tareas de escritura orientadas a desarrollar la reflexión pedagógica, pues así no solo se pone en juego el potencial epistémico y de aprendizaje que esta tiene, sino además se intenciona el pensamiento metacognitivo, mediante un análisis permanente de evaluación y valoración del ejercicio profesional docente propio y de otros.

Es así como la escritura de un diario constituye una valiosa experiencia narrativa en la formación profesional de los docentes, pues es un recurso que permite andamiar distintos tipos y niveles de reflexión pedagógica. Sin embargo, la reflexión no es una habilidad que surja espontáneamente, por lo tanto, se debe enseñar y modelar, y para ello es preciso intencionar las diversas actividades que se diseñen en torno a ella.

En este sentido, es de mucha utilidad modelar la escritura para la reflexión pedagógica desde la enseñanza de los géneros que circulan con estos fines, pues gracias a ello es posible relevar patrones de comportamiento discursivo y luego diseñar secuencias didácticas con foco en el proceso y retroalimentación de los textos que producen bajo estas consignas de escritura.

El diario pasa a constituir un buen reflejo de la etapa en la que los practicantes se encuentran y permite revelar aquellos elementos a los cuales les asignan mayor valor. Desde lo particular, un diario de prácticas tempranas destaca aspectos emocionales que no siempre van de la mano ni se contrastan con conocimientos teóricos, sesgando su mirada sobre el fenómeno educacional y sobre cómo conciben la docencia y el rol del profesor. Por esto es necesario intencionar la enseñanza de la reflexión y para ello resulta fundamental contar con géneros discursivos orales o escritos que permitan graduar el desarrollo de esta según los requerimientos disciplinares y profesionales que se necesiten a lo largo de la formación inicial docente.

\section{REFERENCIAS BIBLIOGRÁFICAS}

Anderson, L., \& Krathwohl, D. (2001). A taxonomy for learning, teaching and assessing: A revision of Bloom's taxonomy of educational objectives. New York: Longman.

Ávalos, B. (2002). Docentes para el siglo XXI. Formación Docente: Reflexiones, debates, desafíos 
e innovaciones. Perspectivas, 32(3). Recuperado desde http://www.ibe.unesco.org/fileadmin/ user_upload/archive/Publications/Prospects/ProspectsOpenFiles/pr123ofs.pdf

Barquín, J. (2002). La tutorización de las prácticas y la socialización del futuro profesorado. Revista de Educación, 327, 267-283.

Bathia, V. (2004). Worlds of writing discourse. A genre based view. Sydney: Continuum.

Boud, D. (2001). Using journal writing to enhance reflective practice. In L. M. English \& M. A. Gillen (Eds.), Promoting Journal Writing in Adult Education. New Directions in Adult and Continuing Education (n ${ }^{\circ}$ 90, pp. 9-18). San Francisco: Jossey-Bass.

Cochran-Smith, M., \& Lytle, S. (2009). Practitioner Research for the Next Generation. New York: Teachers College Press.

Correa, E. (2011). La práctica docente: una oportunidad de desarrollo profesional. Revista Perspectiva Educacional, 50(2), 77-95.

Ershler, A. (2003). La narrativa como texto experiencial: incluirse en el texto. En A. Lieberman y L. Miller (Eds.), La indagación como base de la formación del profesorado y la mejora de la educación (pp. 193-208). Barcelona: Octaedro.

Firdyiwek, Y., \& Scida, E. (2014). Reflective course design: An Interplay between pedagogy and technology in a language teacher education course. International Journal of Portafolio, 4(2), $115-131$.

Haas, V. (2011). Una aproximación a las características personales del buen mentor desde las concepciones de los propios estudiantes de práctica final de Educación Básica de la PUCV (Tesis Doctoral). Universidad de Aconcagua, Chile.

Hatton, N., \& Smith, D. (1995). Reflection in teacher education: towards definition and implementation. Teaching \& Teacher Education, 11(1), 33-49.

Holly, M. (1989). Writing to grow. Keeping a personal-professional journal. Heinnemann. Porsttmouth. New Hampshire: Deakin University.

Imbernón, F. (2004). La formación del profesorado. Barcelona: GRAÓ.

Korthagen, F. (2010). La práctica, la teoría y la persona en la formación del profesorado. Revista Interuniversitaria de Formación del Profesorado, 68, 83-102.

Latorre, A. (1996). El Diario como Instrumento de Reflexión del Profesor Novel. Actas del III Congreso de E. F. de Facultades de Educación y XIV de Escuelas Universitarias de Magisterio. Guadalajara: Ed. Ferloprint.

Lindgren, U. (2005). Experiences of beginning teachers in a school-based mentoring programme Sweden. Educational Studies, 31(3), 251-263.

Liston, D., \& Zeichner, K. (1990). Reflective teaching and action research in preservice teacher education. Journal of Education for Teaching, 16(3), 235-254.

McGuire, L., Lay, K., \& Peters, J. (2009). Pedagogy of reflective writing in professional education. Journal of the Scholarship of Teaching and Learning, 9(1), 93-107.

Nguyen, Q. D., Fernández, N., Karsenti, T., \& Charlin, B. (2014). What is reflection? A conceptual analysis of major definitions and a proposal of a five-component model. Medical education, 48(12), 1176-1189.

Prestridge, S. J. (2014). Reflective Blogging as part of ICT Professional Development to Support Pedagogical Change. Australian Journal of Teacher Education, 39(2), 70-86.

Porlán, R., \& Martín, J. (1993). El Diario del Profesor. Sevilla: Diada.

Swales, J. (2000). Genre Analysis. English in Academic and research settings. Cambridge: Cambridge Press.

Scale, P. (2013). Teaching in the lifelong learning sector. Londres: McGraw-Hill.

Schön, D. (1998). El profesional reflexivo; cómo piensan los profesionales cuando actúan. Barcelona: Paidós.

Tardif, M. (2004). Los saberes del docente y su desarrollo profesional. Madrid: Editorial Narcea.

Tezanos, A. (2007). Formación de Profesores: una reflexión y una propuesta. Revista Pensamiento 
Estudios Pedagógicos XLIII, N²: 163-178, 2017

ESCRITURA PARA LA REFLEXIÓN PEDAGÓGICA: ROL Y FUNCIÓN DEL DIARIO DEL PROFESOR EN FORMACIÓN EN LAS PRÁCTICAS INICIALES

\section{Educativo, 41(2), 57-75.}

Valles, M. (2000). Técnicas cualitativas de Investigación Social. Reflexión metodológica y práctica profesional. Madrid: Síntesis.

Van Manen, M. (1977). Linking ways of knowing with ways of being practical. Curriculum Inquiry, 6, 205-228.

Zabalza, M. (2004). Diarios de clase. Un instrumento de investigación y desarrollo profesional. Madrid: Narcea.

Zabalza, M.A. (2011). El Prácticum en la formación universitaria: estado de la cuestión. Revista de Educación, 354, 21-43.

Zeichner, K. (1986). Preparing reflective teachers: An overview of instructional strategies which have been employed in preservice teacher education. International Journal of Educational Research, II, 565-575. 